\title{
Premières occupations de la montagne sur les versants du col d'Anterne (2 $257 \mathrm{~m})$
}

\section{Pierre-Jérôme Rey}

\section{OpenEdition Journals}

Édition électronique

URL : http://journals.openedition.org/adlfi/1523

ISSN : 2114-0502

Éditeur

Ministère de la culture

Référence électronique

Pierre-Jérôme Rey, «Premières occupations de la montagne sur les versants du col d'Anterne (2 257

m) », ADLFI. Archéologie de la France - Informations [En ligne], Rhône-Alpes, mis en ligne le 01 mars 2008, consulté le 03 mai 2019. URL : http://journals.openedition.org/adlfi/1523

Ce document a été généré automatiquement le 3 mai 2019.

(C) Ministère de la Culture et de la Communication, CNRS 


\title{
Premières occupations de la montagne sur les versants du col d'Anterne (2 $257 \mathrm{~m})$
}

\author{
Pierre-Jérôme Rey
}

Identifiant de l'opération archéologique : 9781

Date de l'opération : 2008 (PT)

1 Cette opération de prospection assortie de sondages a pour but de documenter l'occupation humaine d'une micro-région de la montagne alpine autour du col d'Anterne et de la montagne de Pormenaz. Dans une zone encore peu connue sur le plan archéologique, il s'agit de retrouver et de dater des témoins des premiers peuplements, afin d'aborder la dynamique chronologique et verticale du peuplement, ainsi que les évolutions des systèmes économiques. Ces recherches s'intègrent au programme ANR Pygmalion (direction F. Arnaud, CNRS-Edytem), dont le secteur d'Anterne Pormenaz constitue l'une des quatre zones ateliers. Dans ce cadre, les études coordonnées des sédiments du lac d'Anterne (F. Arnaud et C. Giuet-Covex, Edytem), de remplissages de tourbières (F. David, univ. Aix-Marseille-Cerege et C.Delhon, CNRS-Cepam) et de séquences pédo-sédimentaires (J. Poulenard, INRA-Carrtel) vont permettre de constituer un robuste référentiel paléo-environnemental, indispensable pour contextualiser les données archéologiques.

2 Les premiers résultats montrent une importante pollution au plomb au cours de l'Antiquité qui atteste d'activités liées au travail du métal à proximité, ainsi que d'importantes modifications du couvert végétal à partir du Bronze moyen.

Le secteur considéré a déjà été parcouru en 2005 dans le cadre d'un programme de prospection coordonné par M. Oberkampf et J. Serralongue (SDA Haute-Savoie). Si aucun élément de chronologie n'a pu être apporté, ces premiers travaux ont révélé plusieurs structures pastorales en pierres plus ou moins démantelées. 
4 La méthode mise en oeuvre reprend en partie l'approche développée à grande échelle de 2003 à 2007 autour du Petit-Saint-Bernard : multiplication des petits sondages manuels dans les secteurs de piégeage sédimentaire (plats ou en légère cuvette), description systématique et normalisée des séquences pédo-sédimentaires holocènes. Toute la hauteur des versants est investiguée afin de permettre l'approche des systèmes de remues saisonnières. Compte tenu de leur faible nombre, les structures en pierres sont également abordées par la réalisation de tranchées de sondage.

5 Les prospections préliminaires ont été développées aux alentours de Servoz, et à partir du versant sud du col d'Anterne sur les itinéraires de Plaine-Joux à Alfred Wills, de Moëde à Villy et sur les flancs nord-ouest, nord et nord-est de la montagne de Pormenaz. Quarante-trois sondages ont été entrepris dont deux sont restés inachevés.

6 En basse altitude, les sondages se sont concentrés sur le site des Gures qui présentait les caractéristiques topographiques d'un important site de hauteur (grande surface, fortes défenses naturelles et implantation proche d'un axe de communication important). Des murs arasés de grande largeur, pouvant appartenir à plusieurs enceintes emboîtées y ont été signalés depuis le XIX $\mathrm{e}$. Dans les années 80 , des ramassages à la suite d'un débroussaillage ont livré un petit lot de céramiques du Bas-Empire et un gros fragment de vase en pierre ollaire.

7 Dix-huit sondages ont été effectués sur le plateau intermédiaire situé dans la moitié est du site et quatre sondages sur le pointement sommital dans une série de bâtiments quadrangulaires arasés. Dans seulement quatre profils, il a été possible d'observer des niveaux archéologiques très pauvres, caractérisés par un sédiment teinté de gris et par la présence de pierres et de charbons de bois. S'ils ne sont vraisemblablement pas tous contemporains, les rares éléments mobiliers recueillis (petits fragments de fer rouillés sur le plateau est et un tesson de céramique à revêtement argileux sur le sommet) ne semblent pas être antérieurs à l'âge du Fer. Une série de dates radiocarbones est en cours pour préciser le calage chronologique des niveaux d'occupation observés.

8 Les résultats de l'année 2008 n'apportent aucun élément pour la datation des murs. Des sondages complémentaires sont à prévoir pour achever l'évaluation du site, mais les premiers constats surprennent par la rareté des vestiges antiques et l'absence d'élément protohistorique, surtout en regard de la dimension des supposées enceintes. L'hypothèse d'un site refuge édifié au cours du Bas-Empire, non occupé en permanence et rarement utilisé, pourrait expliquer ces particularités. Une tourbière de basse altitude, partiellement enfouie sous des colluvions de pente, a été repérée sur le plateau est des Gures.

9 En altitude, les recherches se sont concentrées sur un nombre limité de secteurs. Les sondages en plein air à l'écart des sites visibles ou connus n'ont pas donné de résultats. Cette approche extensive reste incontournable pour le plateau d'Anterne où aucune structure ruinée de type pastoral n'a été observée.

10 Sur la montagne de Pormenaz les résultats archéologiques acquis en altitude durant la campagne 2008 proviennent de deux sites à structures apparentes: le Laouchet et le Plane.

11 Au Plane deux enclos subquadrangulaires d'une vingtaine de mètres de diamètre, sont associés à plusieurs petits bâtiments ruinés dont l'un montre encore un toit de tavaillons incomplètement effondré. Les sondages livrent peu d'informations et présentent généralement des séquences peu dilatées. Seul le sondage 5 plaqué contre la paroi 
extérieure de l'un des bâtiments a livré quelques éléments céramiques subcontemporains dans un niveau archéologique dont la fouille n'a pu être achevée en 2008. Le paysage environnant ne révèle pas d'autres structures pour l'instant.

Au Laouchet, devant l'absence de résultats de trois sondages en plein air, les recherches se sont concentrées sur deux structures en pierres. Installé sur le bord d'un petit plateau surmontant une tourbière, le Laouchet 2 se présente comme un bâtiment de $5 \mathrm{mx} 7 \mathrm{~m}$, flanqué d'une cellule plus petite sur le flanc ouest et d'une sorte de petit abri individuel du côté est, vraisemblablement construit plus tardivement. Une tranchée pratiquée à travers un mur du bâtiment principal, montre une stratigraphie très simple de moins d'un mètre d'épaisseur. Sur le substrat rocheux fortement altéré, on observe un premier niveau archéologique mal conservé et quasi dépourvu de vestiges. Quelques fragments de métal rouillé semblent écarter une datation antérieure à l'âge du Fer. Cette couche est surmontée d'un dépôt nettement plus sombre et plus graveleux, contenant parfois des interstratifications charbonneuses, qui est vraisemblablement lié à la dernière occupation de la structure. Un épais niveau de démantèlement présentant d'importantes traces d'incendie surmonte les niveaux d'occupation et livre de rares vestiges mobiliers. Il s'agit de quelques éléments métalliques très fortement corrodés, d'une monnaie indéterminable et d'un tesson à dégraissant millimétrique très altéré. La datation de la dernière phase d'occupation recouvre provisoirement un large intervalle: du Moyen Âge à la période moderne. Cette fourchette pourra être précisée par des datations ${ }^{14} \mathrm{C}$.

13 Situé à une trentaine de mètres plus à l'ouest à l'extrémité d'un petit promontoire, le Laouchet 5 est une accumulation de pierres à peine visible, en forme de fer à cheval de $5 \mathrm{~m}$ de diamètre environ. Une coupe pratiquée à travers la structure montre que le niveau de pierres est mince et très faiblement enfoui. Un petit foyer à plat en cours de datation a été observé au centre de la structure, associé à une fine couche gravillonneuse qui s'insère sous le niveau de démantèlement de la couronne de pierres. Ce type de structure trouve des comparaisons datées de la Protohistoire dans les Alpes du Sud. Il pourrait s'agir d'un petit enclos ou d'une cabane de berger. Plusieurs autres structures en pierre extrêmement arasées ont été repérées à proximité. Le Laouchet présente donc une remarquable concentration de structures en pierres parfois extrêmement peu visibles dans le paysage.

Sur la question de la dynamique pédo-sédimentaire holocène, des séquences à sol enfoui ont été observées en basse comme en haute altitude. Des indices de la présence de sols bruns fersiallitiques peu marqués et assez mal conservés ont été rencontrés en fond de vallée aux Gures. En altitude au-dessus de $1900 \mathrm{~m}$ on ne retrouve pas clairement les sols à horizons blanchis superficiels, très fréquents dans le secteur du Petit-Saint-Bernard. Sur substrat morainique ou sur roches cristallines (Le Laouchet, Le Plane), les séquences observées sont soit fortement perturbées par les activités anthropiques, soit tronquées et peu informatives. Sur substrat calcaire recouvert de fins dépôts de colluvions se développent des sols particuliers, bien représentés dans certaines séquences d'Anterne (Anterne 4, 5, et 10). On observe dans ces trois cas la présence d'au moins un lit de tourbe intercalé et recouvert de colluvions grossières ou d'alluvions torrentielles et deux des séquences présentent en outre une récurrence des horizons de pédogenèse superficiels. S'il est encore tôt pour tirer des hypothèses générales de ces quelques constats, l'observation de récurrences entre les profils et la présence d'éléments datables dans ces stratigraphies offrent d'intéressantes perspectives pour la reconstitution des évolutions du paysage, ainsi que pour la datation des principales phases d'incisions, importante pour 
évaluer la fiabilité des dates ${ }^{14} \mathrm{C}$ obtenues sur les macro-restes issus des sédiments lacustres.

15 La campagne 2009, prévue sur deux mois et une semaine, aura pour objectif la poursuite des recherches de terrain avec le maintien d'une approche extensive sur l'ensemble de la zone de d'étude et un travail plus approfondi dans deux secteurs prioritaires :

16 - les Gures, de manière à achever l'évaluation par sondages du sommet et du plateau ouest ; par ailleurs la topographie générale des structures et des carrières de pierres sera réalisée au GPS ;

17 - le Laouchet, afin de disposer d'une vision complète d'un site probablement pastoral, utilisé sur une longue durée.

REY Pierre-Jérôme

\section{INDEX}

operation Prospection thématique (PRT)

Thèmes : alluvion, céramique du fer, céramique romaine, charbon de bois, colluvion, édifice, enceinte, enclos quadrangulaire, foyer, incendie, lac, métallurgie, monnaie, montagne, objet métallique, occupation du sol, pastoralisme, plomb, site de hauteur, tourbière

Index chronologique : âge du Fer, Empire romain, Moyen Âge, Temps Modernes

Index géographique : Rhône-Alpes, Haute-Savoie (74), Servoz

\section{AUTEURS}

PIERRE-JÉRÔME REY

BEN 